\title{
Tracking donor RBC survival in premature infants: agreement of multiple populations of biotin-labeled RBCs with Kidd antigen-mismatched RBCs
}

\author{
John A. Widness' ${ }^{1}$, Demet Nalbant ${ }^{1}$, Nell I. Matthews ${ }^{3}$, Ronald G. Strauss ${ }^{1,2}$, Robert L. Schmidt ${ }^{1}$, Gretchen A. Cress ${ }^{1}$, \\ Miriam Bridget Zimmerman ${ }^{4}$ and Donald M. Mock ${ }^{3}$
}

BACKGROUND: Anemia, a common condition among critically ill premature infants, is affected by red blood cell (RBC) survival (RCS). We hypothesized that transfused allogeneic Kidd antigen-mismatched RBCs would demonstrate the same concurrent RCS tracking as RBCs multilabeled at separate, discrete low densities with biotin (BioRBCs).

METHODS: Allogeneic RBCs from adult donors were labeled at four biotin densities, mixed, and transfused into 17 anemic premature infants. Nine of the donors and neonates were Kidd antigen mismatched. Serial posttransfusion blood samples were assayed for up to 8 wk by flow cytometry to track the survival of the proportions of Kidd antigen-mismatched and Kidd antigen-biotinylated RBCs.

RESULTS: Using linear mixed modeling to compare results, RCS of the three lowest BioRBC densities was similar to RCS by Kidd antigen mismatch and to one another. RCS of RBCS labeled at the highest BioRBC density was shortened.

CONCLUSION: RCS of different populations of RBCs can be tracked concurrently and reliably using the three lowest BioRBC densities. Although comparable RCS results can be achieved using Kidd antigen mismatches, BioRBCs are preferred for investigating neonatal anemia because biotin labeling of both allogeneic and autologous RBCs is possible.

$S^{\mathrm{c}}$ evere anemia is a common clinical problem among premature and other critically ill infants. An important determinant of anemia is the duration of red blood cell (RBC) survival (RCS) in the circulation. RCS is determined in "cohort" or "population" RBC-labeling experiments (1). Cohort labeling is achieved by in vivo labeling of newly produced RBCs-most commonly by brief exposure to isotopically identifiable iron or glycine-released from the bone marrow all at the same time and therefore of the same age (1-3).

In humans, population RBC labeling is performed ex vivo by labeling RBCs of all ages, with the most common, best known RBC label being ${ }^{51} \mathrm{Cr}$. The same result can be achieved posttransfusion by exploiting intrinsic differences in donor and recipient RBCs. Two of the most common examples of this are donor-recipient differences in major (4) or minor RBC surface antigen expression (5) or in hemoglobin species, e.g., fetal hemoglobin $(\mathrm{HbF})$ vs. adult hemoglobin $(\mathrm{HbA})$ when assayed by flow cytometry using intact cells (4), or by highperformance liquid chromatography using protein hemolysates (6). RBC kinetics may then determined by serially assaying the relative proportions of donor and recipient RBCs (7). These intrinsic methods for determining RCS have the advantage of avoiding the two most common problems resulting in artifactual shortening of RCS, i.e., elution of label from the $\mathrm{RBC}$ surface and $\mathrm{RBC}$ injury from the labeling procedure. Unfortunately, intrinsic methods have the obvious limitation that they can only be applied postallogeneic transfusion and therefore are unsuitable for RCS studies of autologous RBC.

Before 1970s, most methods for determining RBC survival (RCS) relied on radiolabeling of RBCs with ${ }^{51} \mathrm{Cr}$ and ${ }^{32} \mathrm{P}$. At about this time, the use of radioactivity in research became unacceptable for vulnerable study populations including fetuses, infants, children, and pregnant women (8). As a result, studies of the pathophysiology and treatment of anemia in infants and children had few other options for direct measurement of RCS or RBC volume. Fortunately, over the past $20 \mathrm{y}$, measurement of RCS using RBCs labeled with biotin (BioRBCs) has been shown to be practical, reliable, accurate, and safe. The biotin method has the nearly unique capability of being able to concurrently measure RCS for multiple populations of either autologous or allogeneic RBCs. These features of the biotin-labeling method offer important advantages in the investigations of physiology, pathophysiology, and therapy of anemia. These capabilities are particularly important in newborn infants where the determination of RCS is confounded by laboratory phlebotomy loss, growth, and transfusion.

The present study in very low birth weight (VLBW) premature infants compares the tracking of RBCs biotinylated at multiple different biotin densities with RBC tracking based on Kidd antigen differences. On the basis of our prior study of 


\section{Articles | Widness et al.}

RCS of autologous multidensity-labeled RBCs in adults (9), we hypothesized that RBCs labeled with low densities of biotin would exhibit the same RCS as allogeneic-transfused Kidd antigen-mismatched RBCs when concurrently transfused.

\section{RESULTS}

Seventeen premature VLBW infants (nine females) were studied. Gestational age ranged between 26 and $30 \mathrm{wk}$, and birth weight ranged between 0.39 and $1.31 \mathrm{~kg}$ (Table 1). These included 12 singleton (70\%) and 5 twin infants. Body weight at the time of study ranged between 0.37 and $2.21 \mathrm{~kg}$; mean birth weight $z$-score adjusted for gender and singleton or twin status was $-1.24 \pm 1.41(10)$. Mean study age $( \pm$ SD) at the time of the allogeneic study transfusion was $17 \pm 14 \mathrm{~d}$, with a range of 1-45 d. Before study, 10 infants had received between one and three RBC transfusions. All infant study subjects underwent RBC biotinylation followed by posttransfusion RBC tracking. Nine of the 17 had concurrent tracking of donor-recipient Kidd antigen mismatches along with the BioRBCs. For the Kidd mismatches, it was always the donor RBCs, whether positive or negative, that were followed for survival. The nine mismatches include one $\mathrm{Jka}+$, three Jkb+, and two $\mathrm{Jkb}^{-}$donors, and three double mismatches where donor was $\mathrm{Jka}^{-} / \mathrm{Jkb}+$ and baby was $\mathrm{Jka}+/ \mathrm{Jkb}^{-}$.

\section{Tracking of Posttransfusion RCS}

Tracking survival of donor RBCs was performed separately for the two groups of infants, each with a different reference study group. The first analysis included the subgroup of nine infants who had concurrent determinations of Kidd antigens and the four BioRBCs densities (Figure 1a). In this analysis, the Kidd antigen mismatches served as the post hoc analysis reference group (Table 2). With the exception of density of 54 $\mu \mathrm{g} / \mathrm{ml}$ at $2 \mathrm{wk}$, RCS tracking by the three lowest BioRBC densities agreed well and were not statistically different from the RCS tracking by Kidd antigen mismatches for the entire 6-wk study period. By contrast, the highest BioRBC density $(162 \mu \mathrm{g} /$ $\mathrm{ml}$ ) was significantly more rapidly eliminated than Kidd antigen-mismatched RBCs starting at the second week.

The second RCS tracking analysis performed included only the four BioRBC densities followed in all 17-infant study subjects, with the lowest BioRBC density $(6 \mu \mathrm{g} / \mathrm{ml})$ serving as the

Table 1. Premature very low birth weight infant subject and transfusion blood information

\begin{tabular}{|c|c|c|c|c|}
\hline \multirow[b]{2}{*}{ Subject number } & Birth weight $(\mathrm{kg})$ & Postmenstrual age at birth (wk) & Postnatal age at study (d) & Postnatal weight at study $(\mathrm{kg})$ \\
\hline & \multicolumn{4}{|c|}{ Subjects with Kidd antigen mismatches at first transfusion } \\
\hline 1 & 0.83 & 27.86 & 22 & 1.25 \\
\hline 3 & 0.93 & 27.00 & 13 & 1.06 \\
\hline 4 & 0.95 & 26.29 & 5 & 0.92 \\
\hline 6 & 1.00 & 27.00 & 9 & 0.87 \\
\hline $7^{\mathrm{a}}$ & 1.03 & 27.43 & 11 & 1.12 \\
\hline 8 & 1.37 & 28.71 & 10 & 1.32 \\
\hline 9 & 1.40 & 30.14 & 33 & 1.96 \\
\hline Mean & 1.04 & 27.76 & 13.0 & 1.16 \\
\hline $11^{\mathrm{a}}$ & 0.73 & 29.86 & 3 & 0.76 \\
\hline 12 & 0.83 & 27.43 & 15 & 0.98 \\
\hline 13 & 0.86 & 26.71 & 33 & 1.55 \\
\hline 14 & 0.88 & 28.00 & 23 & 1.10 \\
\hline 15 & 0.91 & 27.43 & 45 & 1.76 \\
\hline 16 & 1.08 & 27.43 & 15 & 1.20 \\
\hline 17 & 1.09 & 28.00 & 8 & 0.96 \\
\hline Mean & 0.85 & 27.64 & 17.9 & 1.09 \\
\hline
\end{tabular}

andicates infants studied with fetal hemoglobin-red blood cell tracking. 
post hoc analysis reference group during the 8-wk study period (Figure 1b). Selection of the $6-\mu \mathrm{g} / \mathrm{ml}$ BioRBC density was based on our prior autologous RCS data in adults supporting the current study hypothesis that the lowest BioRBC densities accurately track true RCS (11). For $18 \mu \mathrm{g} / \mathrm{ml}$ BioRBC density, significant post hoc differences with the $6 \mu \mathrm{g} / \mathrm{ml}$ BioRBC reference group were detected at weeks $4,6,7$, and 8 but not at week 5 (Table 3); for $54 \mu \mathrm{g} / \mathrm{ml}$ BioRBC density, statistically significant post hoc differences were observed at week 2 and thereafter except for at week 5. In comparison with the first analysis of the subgroup of nine infants with concurrent Kidd antigen mismatches, the greater number of subjects in the BioRBC analysis $(n=17)$ generated much tighter confidence intervals (Tables 2 and 3 ) and greater sensitivity in detecting post hoc statistically significant differences in the ratio of the 6 $\mu \mathrm{g} / \mathrm{ml}$ reference group relative to the three other BioRBC density differences. However, the differences are so small that they are unlikely to limit the utility of these densities for investigating RCS. For $18 \mu \mathrm{g} / \mathrm{ml}$ BioRBC density, differences were $<10 \%$ and for $54 \mu \mathrm{g} / \mathrm{ml}$ BioRBC density, differences were $<20 \%$. By
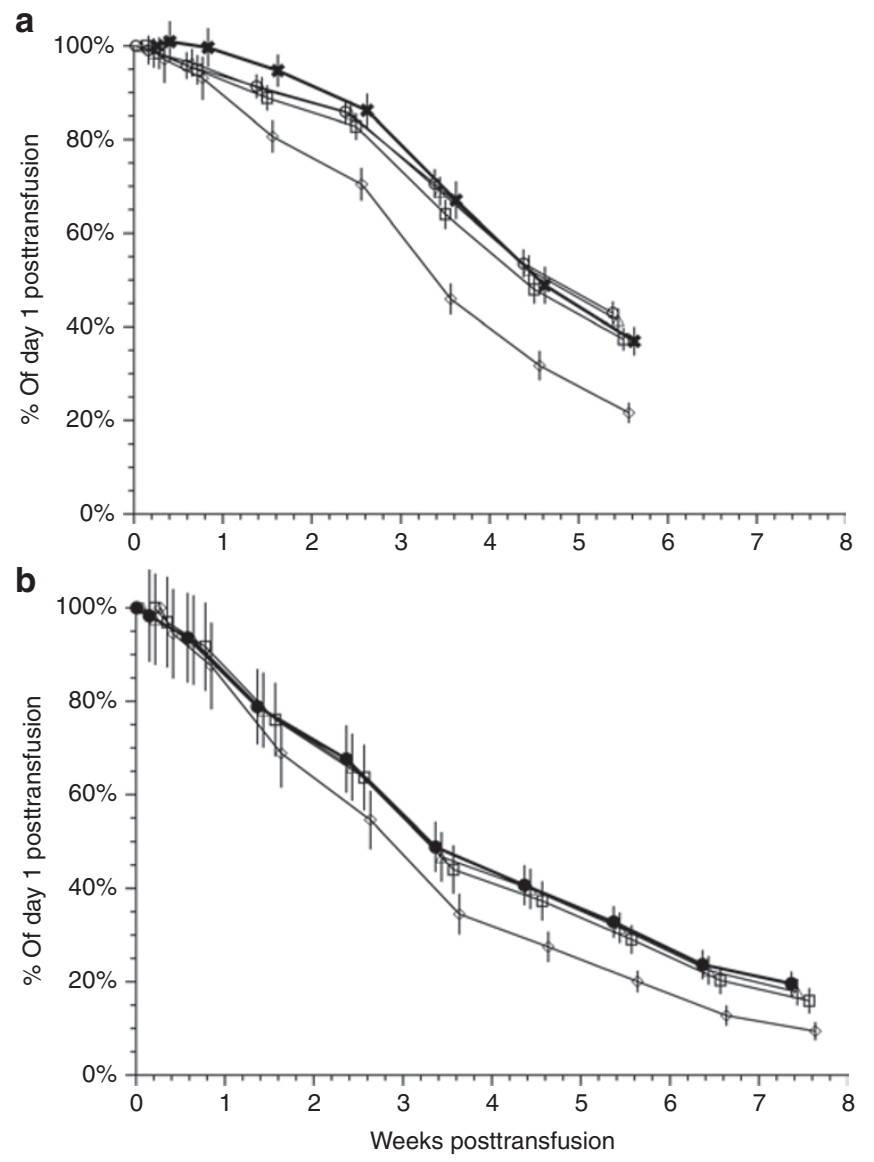

Figure 1. Comparison of mean $( \pm S E M)$ red blood cell $(R B C)$ survival tracking using: (a) Kidd antigen donor-recipient mismatches as the reference control vs. the four different BioRBC densities $(n=9)$ and $(\mathbf{b})$ the lowest BioRBC density as the reference control vs. the three higher BioRBC densities $(n=17)$. The time of study for panel $\mathbf{a}$ is shorter because only data before the second RBC transfusion are included. $x$, Kidd Antigen (Ref); open circles, BioRBC $6 \mu \mathrm{g} / \mathrm{ml}$; open triangles, BioRBC $18 \mu \mathrm{g} / \mathrm{ml}$; open squares, BioRBC $54 \mu \mathrm{g} / \mathrm{ml}$; open diamonds, BioRBC $162 \mu \mathrm{g} / \mathrm{ml}$. contrast, for the $162 \mu \mathrm{g} / \mathrm{ml}$ BioRBC density, the differences were $>10 \%$ by week 2 and were $>40 \%$ by week 7 . These observations provide evidence of a substantial shortening of RCS, possibly due to RBC damage by biotinylation; such an artifact will potentially lead to misleading conclusions when used to compare survival in different RBC populations.

To determine if there were differences in the nine infants included in the first group compared with the eight additional infants included in the second group, a subgroup analysis was performed. Comparisons performed included birth weight, gestational age at birth, postnatal age, gender, and singleton birth. No significant differences were detected (Table 1).

\section{Tracking of Kidd Antigen Double Mismatches and HbF Changes in Selected Infants}

Four infants had unique RCS tracking data following their initial RBC transfusion (Figure 2). For two infants, there was a donor-recipient Kidd antigen double mismatch (i.e., donor RBCs typed as Jka- $\mathrm{Jkb}^{+}$and recipient RBCs typed as $\mathrm{Jka}^{+} \mathrm{Jkb}^{-}$) such that donor RBCs could be tracked by both $\mathrm{Jka}^{-}$and $\mathrm{Jkb}^{+}$ Kidd antigens. Both infants demonstrated concordance in the tracking using both the Kidd antigens before their second RBC transfusion, 35 and $10 \mathrm{~d}$ after their first transfusion (Figure 2a and b). The other two infants had serial $\mathrm{HbF}^{-} \mathrm{RBC}$ measurements tracked following their first transfusion. In both infants, there was agreement between tracking of $\mathrm{HbF}^{-} \mathrm{RBC}$ and the three lowest BioRBC densities (Figure $2 \mathrm{c}$ and d). Again, the heaviest BioRBC density declined more rapidly.

\section{DISCUSSION}

Results of the present study support our hypothesis that RBCs biotinylated at low densities have normal posttransfusion survival compared with the survival of nonbiotinylated, Kidd antigen-mismatched RBCs. By contrast, the RBCs biotinylated at the heaviest density $(162 \mu \mathrm{g} / \mathrm{ml})$ have substantially shortened the long-term RCS, possibly due to biotinylation injury. These conclusions are supported both by comparison with unlabeled RBCs detected by Kidd antigen mismatches and by relative comparison among the four densities of BioRBCs. This conclusion is also in agreement with our previous conclusion concerning shortened survival at higher biotinylation densities in normal adults when in hemodynamic and erythropoietic steady state; the survival of the three lowest BioRBCs densities demonstrated close agreement with one another, and the highest biotinylation density was substantially shortened (9). Although the present study demonstrates comparable minor RBC antigen RCS-tracking results with lightly biotinylated RBCs, the versatility of biotin method in its ability to label both allogeneic and autologous RBCs and to concurrently track multiple RBC populations in all mammalian species are important advantages in the investigations of physiology, pathophysiology, and therapy of anemia (1).

The present RCS-tracking study using BioRBCs, Kidd antigen differences, and $\mathrm{HbF}$ cell change over time extends our previous single BioRBC label infant study (12) by documenting for the first time in VLBW infants that multidensity 


\section{Articles | Widness et al.}

Table 2. Comparison of concurrent posttransfusion red blood cell (RBC) survival of Jka and Jkb RBC antigen donor-recipient mismatches with the four BioRBC densities

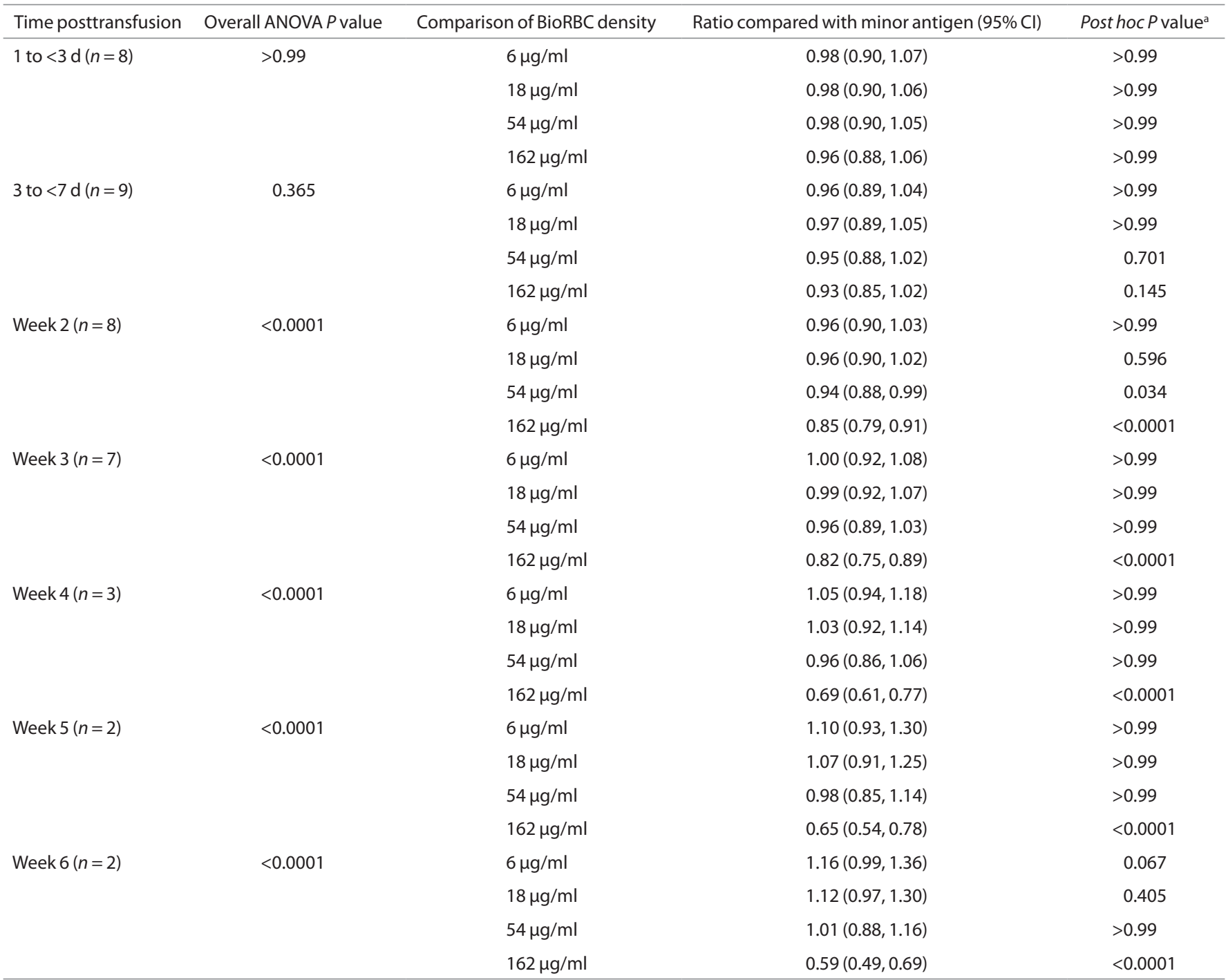

asing Bonferroni's post hoc method.

BioRBCs can be reproducibly and predictably prepared from allogeneic RBCs. Furthermore, concurrent posttransfusion agreement of the tracking of the three lowest BioRBC densities with Kidd antigen-mismatched RBCs indicates that biotinylation at low densities does not alter the survival of transfused RBC. Although there were minor differences among the three lowest BioRBC densities relative to the lowest BioRBC density as the reference (Figure $\mathbf{1 b}$ and Table $\mathbf{3}$ ), the differences observed were small compared with the magnitude of reduced RCS usually deemed to be clinically unimportant. Accordingly, we contend that RBCs biotinylated at the three lowest biotin densities have comparable clinical utility such that they can be used interchangeably to concurrently and accurately track RCS of multiple RBC populations in infants.

The present study findings have substantial implications for the investigation of pathophysiology and treatment of neonatal anemia because acceptable methods for determining posttransfusion RBC kinetics in infants have been limited since 1970 (13). Between 1950 and 1970, RBC labeling with the radionuclide ${ }^{51} \mathrm{Cr}$ became-and continues to be-the only FDA-acceptable RBC-labeling method for assessing RBC kinetics purposes, that is accepted for licensing by the FDA, e.g., licensing of modified RBC transfusion products or storage media $(14,15)$. In the beginning of 1970 , safety concerns regarding radiation exposure of vulnerable populations (fetuses, infants, children, and pregnant women) for research purposes led to the greatly restricted use and eventual abandonment of the use of radioactive RBC labeling in these populations. Consequently, although they commonly receive multiple RBC transfusions, critically ill VLBW infants have not been definitively studied using RBC kinetic methods. As demonstrated in the present study, the current generation of highly sensitive and accurate flow cytometers can detect, distinguish, and accurately enumerate different RBC populations using only a few microliters of blood and in only a few seconds of cytometry flow time. These features of flow 
Table 3. Comparison of concurrent posttransfusion red blood cell survival of lowest BioRBC density of $6 \mu \mathrm{g} / \mathrm{ml}$ with the other three progressively greater BioRBC densities

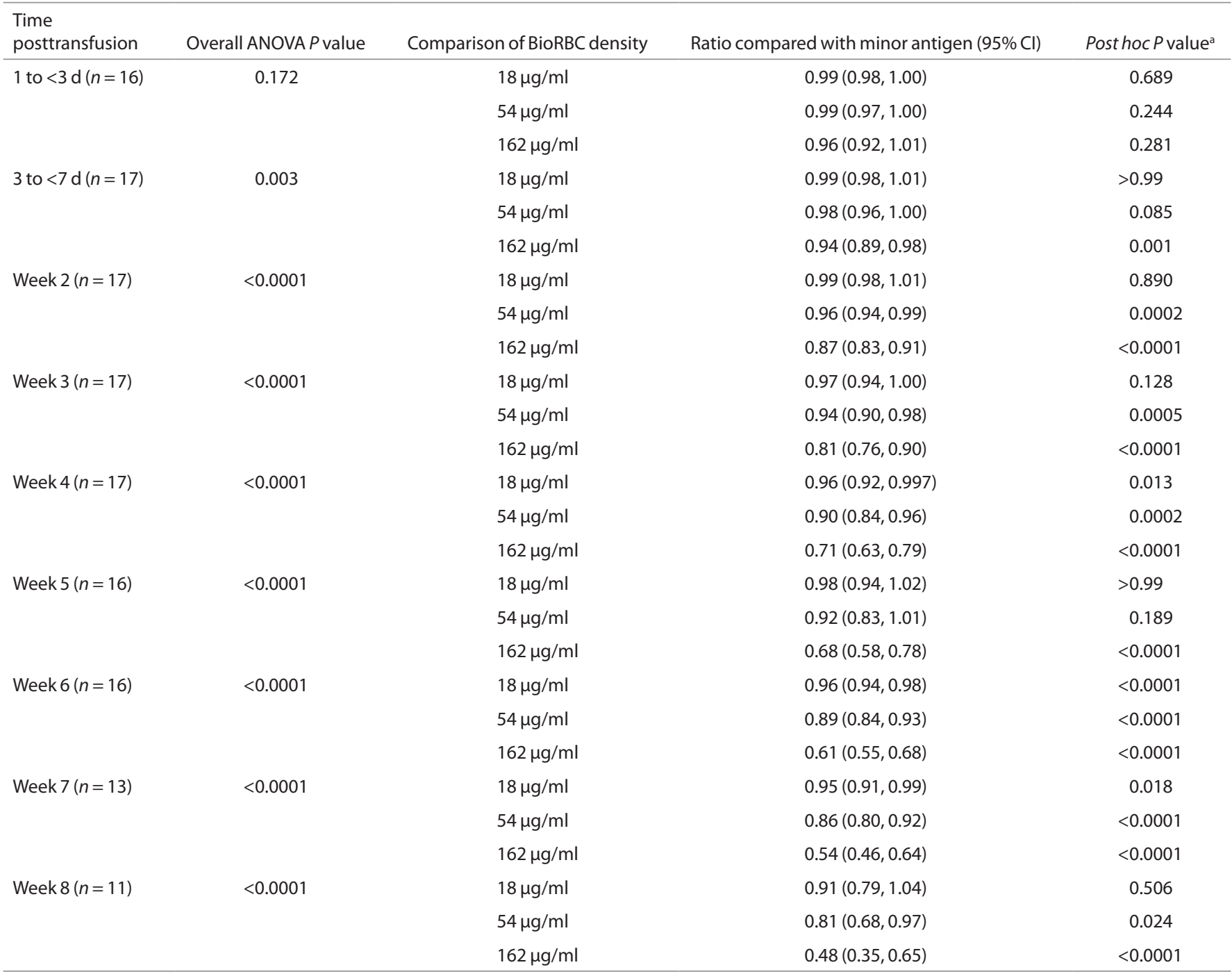

asing Bonferroni's post hoc method.

cytometry offer the potential for resuming RBC kinetic studies in VLBW infants.

As demonstrated in the present study, biotin RBC labeling has the advantage of being able to measure the RCS of multiple RBC populations concurrently in the same individual. Potential applications of multiple RBC population labeling in infants include being able to concurrently track RCS of the same allogeneic donor RBCs stored for different periods in multiply transfused study subjects. Similarly, biotin-labeled autologous RBCs at one density can concurrently be used to track posttransfusion allogeneic donor RBCs labeled at a second biotin density. Although studies in adults have demonstrated the ability to concurrently measure radioactive ${ }^{51} \mathrm{Cr}$ and nonradioactive ${ }^{52} \mathrm{Cr}$ and/or ${ }^{53} \mathrm{Cr}$ (16), these studies are technically challenging and expensive. As a result, multi-Cr-isotopic RBC labeling for concurrently determining RCS of more than one population of RBCs has rarely been applied.
As compared with ${ }^{51} \mathrm{Cr}$-labeled RBCs, tracking of biotinlabeled RBCs can be accurately followed for longer periods of time. In normal healthy adults studied under steadystate erythropoiesis, BioRBCs can be accurately quantitated through the normal $120 \mathrm{~d}$ RBC life span (9). As demonstrated in the present study, this advantage also applies to other flow cytometry cell enumeration procedures in which there are intrinsic differences between donor and recipient RBCs, e.g., in $\mathrm{RBC}$ antigens or in hemoglobin species ( $\mathrm{HbF}$ and $\mathrm{HbA}$ ). Because ${ }^{51} \mathrm{Cr}$ undergoes both radioactive decay and variable $\mathrm{RBC}$ loss-ranging from 0.5 to $2.5 \%$ of ${ }^{51} \mathrm{Cr}$ per day as a result of elution and/or vesiculation of $\mathrm{RBC}$ membrane and intracellular hemoglobin (17) - RCS of ${ }^{51} \mathrm{Cr}$-labeled RBCs requires correction for both these losses and as a result has only been empirically determined for 35 to $40 \mathrm{~d}$.

From the standpoint of ease of use and feasibility, the biotin RBC-labeling method has both advantages and disadvantages. Advantages include the following: (i) the biotinylating 


\section{Articles | Widness et al.}
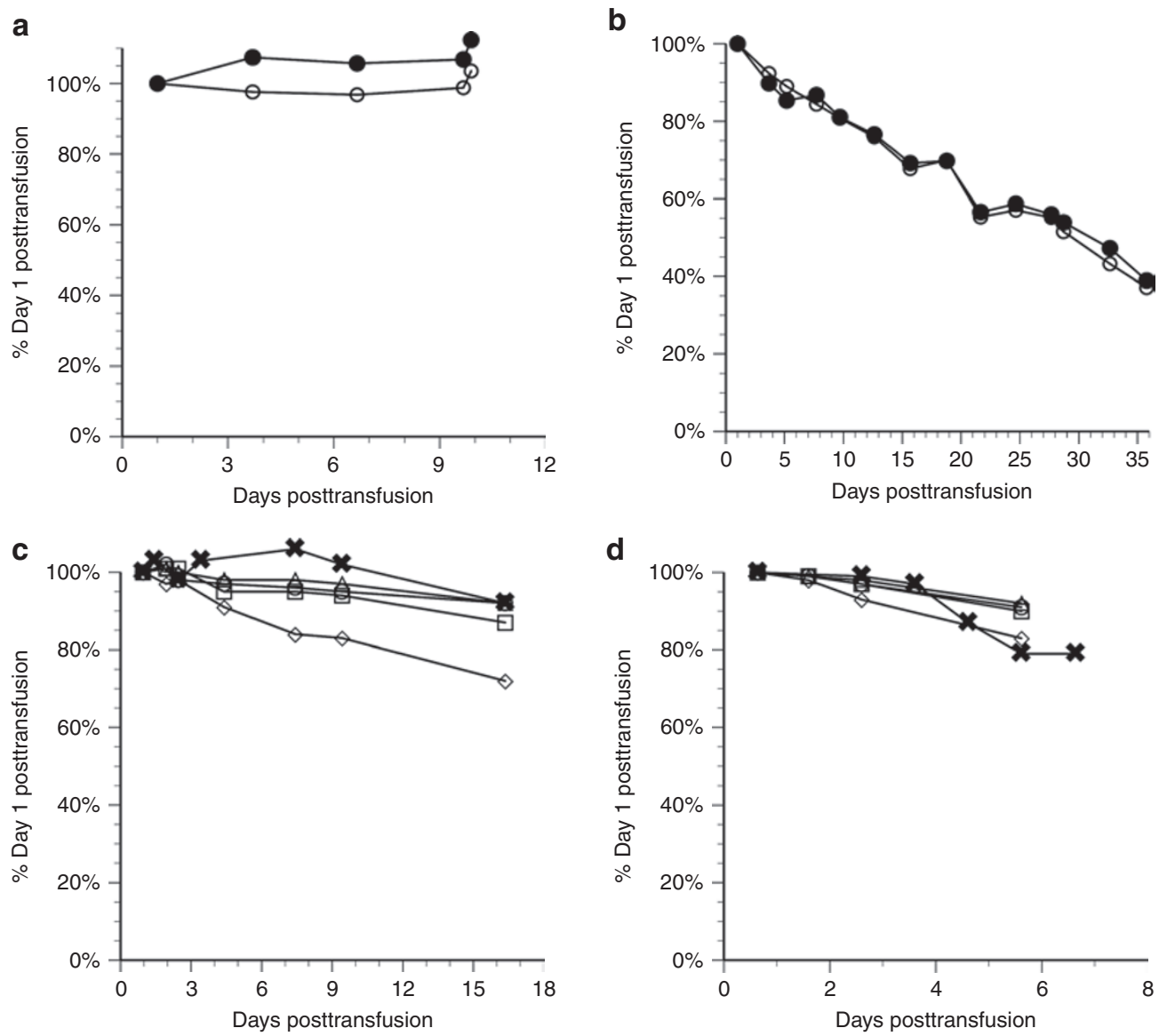

Figure 2. Agreement of fetal hemoglobin ( $\mathrm{HbF}$ ) and Kidd antigen red blood cell (RBC) tracking for selected individual infants following their first RBC transfusion. (a) Infant 11 (see Table 1) HbF-negative cells vs. the four BioRBC densities, (b) infant 7 HbF-negative cells vs. the four BioRBC densities, (c) infant 15 Kidd Jka cells vs. Kidd Jkb cells, and (d) infant 4 Kidd Jka cells vs. Kidd Jkb cells. (a,b) Open circle, Kidd Jka; closed circle, Kidd Jkb; (c,d) x, HbF Neg RBCs; open circles, BioRBC $6 \mu \mathrm{g} / \mathrm{ml}$; open triangles, BioRBC $18 \mu \mathrm{g} / \mathrm{ml}$; open squares, BioRBC $54 \mu \mathrm{g} / \mathrm{ml}$; open diamonds, BioRBC $162 \mu \mathrm{g} / \mathrm{ml}$.

reagents required are inexpensive and commercially available, (ii) the required measurement and synthesis equipment (a flow cytometer and sterile hood) are readily available, and (iii) technician time required for the analysis is modest $(18,19)$. Labeling RBCs with biotin, an essential B vitamin, has been shown to be nontoxic (11) and there have been no adverse clinical consequences other than asymptomatic, transient antibody formation in $15-20 \%$ of the adults $(20,21)$. Disadvantages include the following: (i) the total human experience is limited, and additional safety data are needed and (ii) human studies utilizing BioRBCs require submission of a Food and Drug Administration investigational new drug application.

Tracking of RCS as performed in this study does not permit the quantitative determination of classic RBC kinetic parameters such as mean potential life span or half-life $\left(\mathrm{T}_{50}\right)$. This is because studies in infants include confounding factors not present in adult steady-state studies. These factors include ongoing significant laboratory blood loss (which was not measured in the present study) and hemodilution of labeled RBCs as a result of both $\mathrm{RBC}$ transfusions and growth-related blood volume expansion. When in future studies, RBC enrichment data such as those included in the present study are adjusted for accurate determinations of these confounders, and pharmacodynamic modeling can be applied in deriving meaningful determinations of MPL and $\mathrm{T}_{50}$. When these future RCS data are combined with serial hemoglobin concentration measurements, the rates of RBC production and removal will further extend our understanding of the pathophysiological mechanisms of anemia (22).

In addition to not being able to derive meaningful MPL and $\mathrm{T}_{50}$ determinations, the present study has several limitations. First, the study population consisted of a relatively small convenience sample of only 17 study subjects; Kidd antigenmismatch tracking was possible in only about half of these. Second, the 17 infants were a selected group that may not be a representative of all VLBW infants for all RBC transfusions these infants receive. With the exception of slowly evolving anemia for which all study subjects received nonemergent $15 \mathrm{ml} / \mathrm{kg}$ packed RBC transfusions, infants in the current study were clinically stable at the time of and following transfusion. Third, discrepancies in the changes in Kidd antigen differences and $\mathrm{HbF}$ RBC (but not BioRBC tracking) over time can only be accurately tracked until a second RBC transfusion is administered. Finally, the study was limited to the prehospital discharge period, cutting short the time when BioRBC enrichment can be reliably measured, i.e., $>0.06 \%$ of total RBCs (9). 
In summary, the present study documents that population labeling of donor RBCs with multiple low densities of biotin can be used to accurately track concurrent RCS, i.e., biotin densities of 6,18 , and $54 \mu \mathrm{g} / \mathrm{ml}$. This conclusion is supported by the results demonstrating close agreement in concurrently tracked nonlabeled RBCs using Kidd antigen differences and multidensity-labeled BioRBC populations. This conclusion is also supported by our previous observations in adults (9). The present study lays the groundwork for subsequent studies of survival of autologous RBCs, allogeneic RBCs and both simultaneously in neonates as well as other vulnerable populations including pregnant women, fetuses, infants, and children. RCS studies in the latter three groups are particularly challenging because confounding factors such as phlebotomy loss, growth, and transfusion commonly complicate the determination of long-term RCS. Future studies of RCS in these never before studied, critically ill infants could shed light on new mechanisms contributing to neonatal anemia. Such studies are also likely to be important in developing a rational selection of RBC transfusion therapy (autologous vs. allogeneic RBCs; fresh vs. stored RBCs) and in evaluating the effectiveness of other therapies for neonatal anemia including delayed cord clamping, umbilical cord milking, erythropoietin treatment, and other future therapies.

\section{METHODS}

The study was approved by the University of Iowa Committee on research on human subjects (performance site) and by the Institutional Review Board at the University of Arkansas for Medical Sciences (analysis site). This included approval for administering Kidd antigen-mismatched RBCs. Informed written parental consent was obtained.

\section{Study Subjects}

A prospective, convenience sample of 122 eligible newborn infants included those with $<31$-wk gestation for whom survival was anticipated and those who were scheduled to receive a clinically indicated $\mathrm{RBC}$ transfusion. Infants who had received a prior RBC transfusion were not excluded. Infants with congenital anomalies were excluded.

\section{Study Protocol}

Following enrollment, a pretransfusion-discarded blood sample was analyzed to determine the Kidd antigen expression of study subjects. When a transfusion was expected, fresh $(<7 \mathrm{~d}$ old) blood bank donor RBC units stored in AS3-preservative solution (Leukotrap RC System; Medsep, Covina, CA) were analyzed similarly for Kidd antigen expression. Kidd antigen mismatches were defined as differences in Kidd antigen expression between infant study subjects and adult donor blood. Blood bank RBC units identified as Kidd antigen mismatched were maintained in the blood bank until a clinically indicated RBC transfusion was ordered. Occasionally, this mismatch occurred by chance before the screening procedure could select a confirmed donor mismatch. Both qualitative and quantitative Kidd antigen testing was performed using the basic procedure described below.

Blood bank RBC units identified as Kidd antigen mismatched were maintained in the blood bank until a clinically indicated RBC transfusion was ordered. Once ordered, the designated AS-3 donor unit underwent volume reduction by centrifugation to a packed red cell volume of $\sim 0.85$. Standard practice in our neonatal intensive care unit is to transfuse with $15 \mathrm{ml} / \mathrm{kg}$ packed RBCs. In this study, the first $11 \mathrm{ml} / \mathrm{kg}$ of the transfusion was administered over 3-4h. The remaining allogeneic-packed RBCs (minimum of $4 \mathrm{ml} / \mathrm{kg}$, plus $2.8 \mathrm{ml}$ to allow adequate volume for blood culture and hematological analysis) were brought to the research laboratory and four equal aliquots of $\sim 1.7 \mathrm{ml} / \mathrm{kg}$ each were individually labeled at each of the four discrete biotin densities, as previously described (9) and briefly summarized below. This BioRBC dose results in an enrichment of $2.0-2.5 \%$ for each BioRBC density.

Following the last wash step of biotinylation procedure described below, the supernatant fractions were removed and equal volumes of the four packed BioRBC populations, each with hematocrits of $75-85 \%$, were combined, mixed, and then passed through an 18-micron filter (Hemo-Nate; Utah Medical Products, Midvale, UT) to remove microaggregates. An aliquot of the labeled RBC mixture was cultured aerobically for bacteria (BD Bactec Plus Aerobic/F; Becton Dickinson, Sparks, MD). Within a few minutes after completing the $11 \mathrm{ml} / \mathrm{kg}$ clinical transfusion, the final $4 \mathrm{ml} / \mathrm{kg}$ of BioRBCs were infused over $10 \mathrm{~min}$ to complete the $15 \mathrm{ml} / \mathrm{kg}$ transfusion.

Beginning at $24 \mathrm{~h}$ after the $4 \mathrm{ml} / \mathrm{kg}$ BioRBC transfusion and ending at hospital discharge, leftover anticoagulated whole blood from clinically ordered laboratory testing was salvaged twice weekly for quantitative analysis of the proportions of BioRBCs and Kidd antigens present as described below.

\section{Biotinylation of RBCs}

The method of biotin-labeling RBCs has been previously described (11). In brief, RBCs were washed with a carbonate-buffered dextrosesodium phosphate wash solution to remove plasma proteins and resuspended at a hematocrit of $25 \%$. For the hematocrit adjustment and for the BioRBC count before transfusion, hematological analysis was performed using the Sysmex XE-2100 (Sysmex, Kobe, Japan). Four equal volume aliquots of RBCs were then incubated with four threefold serial dilutions of the biotinylating reagent, sulfosuccinimidobiotin (sNHS-biotin; Pierce Chemical, Rockford, IL), yielding final concentrations of $6,18,54$, and $162 \mu \mathrm{g}$ of sNHS-biotin/ml RBCs. The threefold density differences in the four densities of BioRBCs were previously demonstrated to provide complete separation from unlabeled RBCs and from the other biotin-labeled RBC populations. Separation of the four BioRBC peaks enhances accuracy in the quantitation of the populations by avoiding the need to correct for peak overlap. Maintaining the separate individual peaks as close together as possible maximizes the number of useful RBC populations.

\section{Quantitative Flow Cytometric RBC Analysis}

BioRBC. For each whole blood sample, the proportion of each population of biotinylated RBCs was determined by flow cytometric enumeration, as previously described $(9,23,24)$. In brief, labeled RBCs were enumerated by flow cytometry using the FACSCalibur (BD Biosciences, San Jose, CA). Enrichment proportions were calculated as the ratio of the number of cells in each of the four manually specified population interval markers relative to the total number of RBCs counted. To enhance accuracy, $1 \times 10^{6}$ total events (i.e., cells) were counted. Under these conditions, the lower limit of quantitation for each BioRBC population is $0.06 \%$ of total RBCs (9).

Kidd RBC Antigen Mismatch. Kidd RBC antigens were selected for the study because: (i) Kidd RBC antigens are reliably separated into distinct flow cytometric peaks using commercially available labeled antibodies and (ii) the differences in donor-recipient antigen expression are predicted to frequently occur because of the relatively large percentage of individuals who either express or lack these antigens (i.e., potential donor-recipient "mismatches" are frequent). With respect to the latter, $47 \%$ of the whites and $91 \%$ of the blacks express the JKa antigen, with 23 and 9\%, respectively, lacking the antigen. For $\mathrm{JKb}, 72 \%$ of the whites and $43 \%$ of the blacks express the antigen with 28 and $57 \%$, respectively, lacking the antigen.

For flow cytometric analysis of both $\mathrm{Jka}^{+}$and $\mathrm{Jkb}^{+} \mathrm{RBC}$, triplicate aliquots of $5 \mu \mathrm{l}$ of the pre- and posttransfusion samples were washed to remove plasma proteins, following a similar protocol as for BioRBCs $(9,24)$. RBCs were suspended in $0.3 \mathrm{ml}$ of wash buffer with $2 \%$ BSA and incubated at room temperature overnight along with 5 $\mu \mathrm{l}$ of anti-Jka or anti-Jkb primary antibody (Immucor, Norcross, GA). Overnight incubation was done with continuous mixing on a rotating wheel (Roller Drum Model TC-7; New Brunswick Scientific, Edison, NJ). Excess antibody was removed in two washes before subsequent 
incubation with a Alexa Fluor 488-conjugated antihuman secondary antibody (H10120; Invitrogen, Carlsbad, CA) at $37{ }^{\circ} \mathrm{C}$ for $1 \mathrm{~h}$. Before flow cytometry analysis, RBCs were washed three times and resuspended in wash buffer plus $2 \%$ BSA that had been prefiltered through a 0.2 micron filter to a final volume of $0.5 \mathrm{ml}$. The relative percentages of Jka antigen or Jkb antigen-positive and Jkb antigen-negative RBCs included under the two manually specified peaks relative to the total number of RBCs were determined from 200,000 gated events. Depending on the subject's Kidd antigen-mismatch status, sometimes the antigen-negative peak was followed.

$H b F^{+}$RBCs. Following allogeneic adult donor RBC transfusion, infant RBCs (that nearly exclusively contain $\mathrm{HbF}$ and are denoted as $\mathrm{HbF}^{+} \mathrm{RBCs}$ ) are easily distinguished by flow cytometry from allogeneic adult donor RBCs (that nearly exclusively contain $\mathrm{HbA}$ ). The percentage of $\mathrm{HbF}^{+} \mathrm{RBCs}$ in blood samples was determined by the Invitrogen Fetal Hemoglobin Test kit (Invitrogen, Camarillo, CA) using FITCconjugated monoclonal antibody directed against $\mathrm{HbF}(25)$. These kits are intended to detect small percentages of $\mathrm{HbF}$ cells in maternal blood. In demonstrating a linear response from 0 to $100 \% \mathrm{HbF}$ cells, we were assured that these kits were reliable in the current study. The manufacturer's procedure was followed except for the following minor modifications. Duplicate $1.5 \mu \mathrm{l}$ whole blood samples $\left(\sim 5 \times 10^{6}\right.$ cells $)$ were fixed in $0.25 \mathrm{ml}$ of $0.05 \%$ glutaraldehyde. RBCs were then permeabilized in $0.2 \mathrm{ml}$ of $0.1 \%$ Triton X-100. Washed and pelleted RBCs were resuspended in $80 \mu$ of phosphate-buffered saline/bovine serum albumin and incubated with $\mathrm{HbF}$ monoclonal antibody for $30 \mathrm{~min}$ at room temperature. RBCs were washed and resuspended in $0.4 \mathrm{ml}$ of $2 \%$ paraformaldehyde before flow cytometric analysis. $\mathrm{HbF}^{+} \mathrm{RBCs}$ were enumerated with at least 100,000 events by a flow cytometry.

\section{Data Handling and Statistical Analysis}

Tracking of RCS survival was performed using linear mixed model analysis. The data used for the RCS tracking of the four different BioRBC populations and the two Kidd antigen-mismatched RBC populations were expressed as a ratio relative to the sample at about $24 \mathrm{~h}$ after the $4 \mathrm{ml} / \mathrm{kg}$ transfusion, as recommended by International Committee for Standardization in Haematology for RCS measurement (17). Because blood samples for RCS tracking of individual infants were not obtained at precisely the same time (see description of left over blood availability above), the ratios at the individual time points were grouped into the following intervals: $1 \mathrm{~d}$ to $<3 \mathrm{~d}, 3 \mathrm{~d}$ to $<7 \mathrm{~d}$, and weekly intervals thereafter (e.g., $7 \mathrm{~d}$ to $<14 \mathrm{~d}$ through $49 \mathrm{~d}$ to $<56 \mathrm{~d}$ ). Multiple data points for a subject within a given interval were treated statistically as replicates. Because the ratios did not have a normal distribution, a natural log transformation was applied to the data before linear mixed model analysis.

Two mixed models were fitted. One compared RCS tracking of Kidd antigens (Jka and Jkb) with the RCS tracking of the four BioRBC densities $(6,18,54$, or $162 \mu \mathrm{g} / \mathrm{ml})$. The other compared RCS tracking of the lowest BioRBC density vs. the three heavier BioRBC densities. The fixed effects in both models included density (or method), time, and density $\times$ time interaction. In both, the test for density $\times$ time interaction corresponds to test whether RCS tracking over time shows nonparallel profiles among the densities and Kidd antigens. In addition to estimating the fixed effects in the mixed model, this analytic method permits selection of the covariance structure that best fits the relationship of the RCS data (i.e., BioRBC densities and Kidd antigens at different times) measured in the same subject. From the fitted models, tests of mean contrasts were performed to compare each of the BioRBC densities with Kidd antigens as the reference (first model) or the three heavier BioRBC densities vs. reference density $6 \mu \mathrm{g} / \mathrm{ml}$ (second model) at each time interval. Because the tracking of RCS for Kidd antigen mismatches in the first model is valid only prior to the times before a second RBC transfusion was administered, time intervals for the data included in this analysis extend only through posttransfusion week 6 while the second model was extended through posttransfusion week 8 . The $P$ values for these tests were adjusted using the Bonferroni post hoc method to account for the number of comparisons. The means of the natural log transformed data were back transformed to obtain the mean ratios for each biotin density and for the Kidd antigen at each time interval. Descriptive summary results are presented as the mean $\pm \mathrm{SD}$. A $P$ value $<0.05$ was considered statistically significant.

\section{ACKNOWLEDGMENTS}

The authors acknowledge the helpful discussions with Robert S. Franco and Peter Veng-Pedersen regarding methodological and theoretical aspects of the present study. We appreciate the many contributions of the clinical laboratory staff led by Mitchell J. Owen and overseen by Matthew D. Krasowski, without which this work would not have been possible. We also acknowledge the many outstanding clinical research contributions of lowa's neonatal nurse research team (Karen Johnson, Sara Scott, and Ruthann Schrock) and the research laboratory team (Earl Gingerich and Jessica Goehring). Mark Hart provided valuable editorial and secretarial assistance. The Sysmex XE-2100 automatic hematology analyzer used in this study was generously provided on an on-loan basis from Sysmex, Kobe, Japan. Finally, we appreciate the willingness of the study subject families in allowing their infants to participate. This trial has been registered at www.clinicaltrials.gov (identifier NCT00731588).

\section{STATEMENT OF FINANCIAL SUPPORT}

This publication was supported in part by US Public Health Service National Institutes of Health (NIH) grant P01 HL046925 (to J.A.W.), by the Thrasher Research Fund 0285-3, and by grant UL1RR024979 from the National Center for Research Resources (to J.A.W.), a part of the NIH. The paper's contents are solely the responsibility of the authors and do not necessarily represent the official views of the $\mathrm{NIH}$, the Thrasher Research Fund, or the Clinical and Translational Science Award (CTSA) Program.

Disclosure: The authors have nothing to disclose.

\section{REFERENCES}

1. Franco RS. The measurement and importance of red cell survival. Am J Hematol 2009;84:109-14.

2. Dancis J, Danoff S, Zabriskie J, Balis ME. Hemoglobin metabolism in the premature infant. J Pediatr 1959;54:748-55.

3. Garby L, Sjoelin S, Vuille JC. Studies on erythro-kinetics in infancy. v. estimations of the life span of red cells in the newborn. Acta Paediatr 1964;53:165-71.

4. Wynn R, Dixon S, al-Ismail SA, et al. Flow cytometric determination of pre-transfusion red cell volume in fetuses and neonates requiring transfusion based on $\mathrm{RhD}+$ dilution by transfused $\mathrm{D}$ - red cells. Br J Haematol 1995;89:620-2.

5. Hudson I, Cooke A, Holland B, et al. Red cell volume and cardiac output in anaemic preterm infants. Arch Dis Child 1990;65(7 Spec No):672-5.

6. Phillips HM, Holland BM, Abdel-Moiz A, et al. Determination of red-cell mass in assessment and management of anaemia in babies needing blood transfusion. Lancet 1986;1:882-4.

7. Klein HG, Anstee DJ. Appendix 7. Red cell survival methods based on antigenic differences between donor and recipient. In: Mollison's Blood Transfusion in Clinical Medicine. Oxford, UK: Blackwell Publishing, 2005:850851.

8. Diekema DS. Conducting ethical research in pediatrics: a brief historical overview and review of pediatric regulations. J Pediatr 2006;149:Suppl $1:$ S3-11.

9. Mock DM, Matthews NI, Zhu S, et al. Red blood cell (RBC) survival determined in humans using RBCs labeled at multiple biotin densities. Transfusion 2011;51:1047-57.

10. Arbuckle TE, Wilkins R, Sherman GJ. Birth weight percentiles by gestational age in Canada. Obstet Gynecol 1993;81:39-48.

11. Mock DM, Matthews NI, Zhu S, et al. Red blood cell (RBC) volume can be independently determined in vivo in humans using RBCs labeled at different densities of biotin. Transfusion 2011;51:148-57.

12. Strauss RG, Mock DM, Widness JA, Johnson K, Cress G, Schmidt RL. Posttransfusion 24-hour recovery and subsequent survival of allogeneic red blood cells in the bloodstream of newborn infants. Transfusion 2004;44:871-6.

13. Brugnara C, Platt OS. The neonatal erythrocyte and its disorders. In: Nathan DG, Oski FA, eds. Nathan and Oski's Hematology of Infancy and Childhood. Philadelphia, PA: WB Saunders, 2003:19-55. 
14. Dumont LJ, AuBuchon JP. Evaluation of proposed FDA criteria for the evaluation of radiolabeled red cell recovery trials. Transfusion 2008;48:105360.

15. Dumont LJ, AuBuchon JP. BEST Collaborative. Evaluation of proposed FDA criteria for evaluation of radiolabeled red cell recovery trials, 2008. (http://www.fda.gov/ohrms/dockets/ac/08/slides/2008-4355S1-00-index. html).

16. Silver HM, Seebeck M, Carlson R. Comparison of total blood volume in normal, preeclamptic, and nonproteinuric gestational hypertensive pregnancy by simultaneous measurement of red blood cell and plasma volumes. Am J Obstet Gynecol 1998;179:87-93.

17. Recommended method for radioisotope red-cell survival studies. International Committee for Standardization in Haematology. Br J Haematol 1980;45:659-66.

18. Mock DM, Lankford GL, Widness JA, Burmeister LF, Kahn D, Strauss RG. Measurement of red cell survival using biotin-labeled red cells: validation against 51Cr-labeled red cells. Transfusion 1999;39:156-62.

19. Mock DM, Lankford GL, Widness JA, Burmeister LF, Kahn D, Strauss RG RBCs labeled at two biotin densities permit simultaneous and repeated measurements of circulating RBC volume. Transfusion 2004;44:431-7.
20. Cordle DG, Strauss RG, Lankford G, Mock DM. Antibodies provoked by the transfusion of biotin-labeled red cells. Transfusion 1999;39: 1065-9.

21. Mock DM, Widness JA, Strauss RG, Franco RS. Posttransfusion red blood cell (RBC) survival determined using biotin-labeled RBCs has distinct advantages over labeling with (51) Cr. Transfusion 2012;52:1596-8.

22. Freise KJ, Widness JA, Veng-Pedersen P. Erythropoietic response to endogenous erythropoietin in premature very low birth weight infants. J Pharmacol Exp Ther 2010;332:229-37.

23. Mock DM, Matthews NI, Strauss RG, Burmeister LF, Schmidt R, Widness JA. Red blood cell volume can be independently determined in vitro using sheep and human red blood cells labeled at different densities of biotin. Transfusion 2009;49:1178-85.

24. Mock DM, Matthews NI, Zhu S, et al. Red blood cell (RBC) volume can be independently determined in vivo in the sheep using ovine RBCs labeled at different densities of biotin. Transfusion 2010;50:2553-64.

25. Davis BH, Olsen S, Bigelow NC, Chen JC. Detection of fetal red cells in fetomaternal hemorrhage using a fetal hemoglobin monoclonal antibody by flow cytometry. Transfusion 1998;38:749-56. 